\title{
Research on some types of fractional differential equations which can be transformed into separable variables
}

\author{
Chiihuei $\mathrm{Yu}^{*}$ \\ School of Mathematics and Statistics, Zhaoqing University, Guangdong, China
}

\begin{abstract}
Keywords: fractional differential equations, separable variables, Jumarie type of modified Riemann-Liouville fractional derivatives, new multiplication, product rule.
\end{abstract}

\begin{abstract}
In this paper, we study some types of fractional differential equations which can be transformed into separable variables, regarding the Jumarie type of modified Riemann-Liouville fractional derivatives. We use a new multiplication of fractional functions and product rule for fractional derivatives to obtain the solutions of these fractional differential equations. Furthermore, some examples are given to demonstrate our results.
\end{abstract}

\section{Introduction}

Fractional calculus, a popular name used to denote the calculus of non-integer order, is as old as the calculus of integer order as created independently by Newton and Leibniz. In contrast with the calculus of integer order, fractional calculus has been granted a specific area of mathematics only in 1974, after the first international congress dedicated exclusively to it. Before this congress there were only sporadic independent papers, without a consolidated line [21-24]. It is nowadays well established that several real life phenomena are better described by fractional differential equations, where the term fractional, used for historical reasons, refers to derivative operators of any real positive order. Applications of fractional differential equations are commonly found in bioengineering, chemistry, control theory, electronic circuit theory, mechanics, physics, seismology, signal processing and so on ([1-20]). We refer to [8] for an historical perspective on fractional calculus. Unlike standard calculus, there is no unique definition of derivation and integration in fractional calculus. The commonly used definition is the Riemann-Liouville (R-L) fractional derivative [21]. Other useful definitions include Caputo definition of fractional derivative [22], the Grunwald-Letinikov (G-L) fractional derivative [23], and Jumarie's modified R-L fractional derivative [25].

In this paper, we study three types of fractional differential equations which can be transformed into separable variables, regarding the Jumarie type of modified R-L fractional derivatives. We define a new multiplication of fractional functions and use product rule for

\footnotetext{
*Corresponding author:2019013010@zqu.edu.cn
} 
fractional derivatives to solve these fractional differential equations. On the other hand, we give some examples to illustrate the methods used in this paper.

\section{Preliminaries and methods}

At first, the fractional calculus used in this article is introduced below.

Definition 2.1: Suppose that $\alpha$ is a real number and $p$ is a positive integer. Then the modified Riemann-Liouville fractional derivatives of Jumarie type ([25]) is defined by

$$
{ }_{a} D_{x}^{\alpha}[f(x)]=\left\{\begin{array}{cc}
\frac{1}{\Gamma(-\alpha)} \int_{a}^{x}(x-\tau)^{-\alpha-1} f(\tau) d \tau, & \text { if } \alpha<0 \\
\frac{1}{\Gamma(1-\alpha)} \frac{d}{d x} \int_{a}^{x}(x-\tau)^{-\alpha}[f(\tau)-f(a)] d \tau & \text { if } 0 \leq \alpha<1 \\
\frac{d^{p}}{d x^{p}}\left({ }_{a} D_{x}^{\alpha-p}\right)[f(x)], & \text { if } p \leq \alpha<p+1
\end{array}\right.
$$

where $\Gamma(\rho)=\int_{0}^{\infty} t^{\rho-1} e^{-t} d t$ is the gamma function defined on $\rho>0$.

Proposition 2.2 ([26]): Let $\alpha, \beta, c$ be real numbers and $\beta \geq \alpha>0$, then

$$
{ }_{0} D_{x}^{\alpha}\left[x^{\beta}\right]=\frac{\Gamma(\beta+1)}{\Gamma(\beta-\alpha+1)} x^{\beta-\alpha},
$$

and

$$
{ }_{0} D_{x}^{\alpha}[c]=0 .
$$

Definition 2.3 ([27]): The Mittag-Leffler function is defined by

$$
E_{\alpha}(z)=\sum_{k=0}^{\infty} \frac{z^{k}}{\Gamma(k \alpha+1)}
$$

where $\alpha$ is a real number, $\alpha>0$, and $z$ is a complex variable.

Next, a new multiplication of fractional functions is introduced below.

Definition 2.4 ([9]): Suppose that $\lambda, \mu, z$ are complex numbers, $0<\alpha \leq 1, j, l, k$ are non-negative integers, and $a_{k}, b_{k}$ are real numbers, $p_{k}(z)=\frac{1}{\Gamma(k \alpha+1)} z^{k}$ for all $k$. The $\otimes$ multiplication is defined by

$$
\begin{aligned}
p_{j}\left(\lambda x^{\alpha}\right) \otimes p_{l}\left(\mu y^{\alpha}\right)= & \frac{1}{\Gamma(j \alpha+1)}\left(\lambda x^{\alpha}\right)^{j} \otimes \frac{1}{\Gamma(l \alpha+1)}\left(\mu y^{\alpha}\right)^{l}= \\
& \frac{1}{\Gamma((j+l) \alpha+1)}\left(\begin{array}{c}
j+l \\
j
\end{array}\right)\left(\lambda x^{\alpha}\right)^{j}\left(\mu y^{\alpha}\right)^{l},(5)
\end{aligned}
$$

where $\left(\begin{array}{c}j+l \\ j\end{array}\right)=\frac{(j+l) !}{j ! l !}$.

If $f\left(\lambda x^{\alpha}\right)$ and $g\left(\mu y^{\alpha}\right)$ are two fractional functions,

$$
\begin{aligned}
& f\left(\lambda x^{\alpha}\right)=\sum_{k=0}^{\infty} a_{k} p_{k}\left(\lambda x^{\alpha}\right)=\sum_{k=0}^{\infty} \frac{a_{k}}{\Gamma(k \alpha+1)}\left(\lambda x^{\alpha}\right)^{k}, \\
& g\left(\mu y^{\alpha}\right)=\sum_{k=0}^{\infty} b_{k} p_{k}\left(\mu y^{\alpha}\right)=\sum_{k=0}^{\infty} \frac{b_{k}}{\Gamma(k \alpha+1)}\left(\mu y^{\alpha}\right)^{k},
\end{aligned}
$$

then we define

$$
\begin{gathered}
f\left(\lambda x^{\alpha}\right) \otimes g\left(\mu y^{\alpha}\right)=\sum_{k=0}^{\infty} a_{k} p_{k}\left(\lambda x^{\alpha}\right) \otimes \sum_{k=0}^{\infty} b_{k} p_{k}\left(\mu y^{\alpha}\right) \\
=\sum_{k=0}^{\infty}\left(\sum_{m=0}^{k} a_{k-m} b_{m} p_{k-m}\left(\lambda x^{\alpha}\right) \otimes p_{m}\left(\mu y^{\alpha}\right)\right) .
\end{gathered}
$$




\section{Proposition 2.5:}

$f\left(\lambda x^{\alpha}\right) \otimes g\left(\mu y^{\alpha}\right)=\sum_{k=0}^{\infty} \frac{1}{\Gamma(k \alpha+1)} \sum_{m=0}^{k}\left(\begin{array}{c}k \\ m\end{array}\right) a_{k-m} b_{m}\left(\lambda x^{\alpha}\right)^{k-m}\left(\mu y^{\alpha}\right)^{m}$.

Definition 2.6: Let $\left(f\left(\lambda x^{\alpha}\right)\right)^{\otimes n}=f\left(\lambda x^{\alpha}\right) \otimes \cdots \otimes f\left(\lambda x^{\alpha}\right)$ be the $n$ times product of the fractional function $f\left(\lambda x^{\alpha}\right)$. If $f\left(\lambda x^{\alpha}\right) \otimes g\left(\lambda x^{\alpha}\right)=1$, then $g\left(\lambda x^{\alpha}\right)$ is called the $\otimes$ reciprocal of $f\left(\lambda x^{\alpha}\right)$, and denoted as $\left(f\left(\lambda x^{\alpha}\right)\right)^{\otimes-1}$.

Definition 2.7: If $\mathrm{f}(z)=\sum_{k=0}^{\infty} a_{k} z^{k}, g\left(\mu x^{\alpha}\right)=\sum_{k=0}^{\infty} b_{k} p_{k}\left(\mu x^{\alpha}\right)$, then

$$
f_{\otimes \alpha}\left(g\left(\mu x^{\alpha}\right)\right)=\sum_{k=0}^{\infty} a_{k}\left(g\left(\mu x^{\alpha}\right)\right)^{\otimes k}
$$

The following is the major method used in this paper.

Theorem 2.8 (product rule for fractional derivatives) ([9]): If $0<\alpha \leq 1, \lambda, \mu$ are complex numbers, and $f, g$ are fractional functions. Then

$$
\begin{array}{r}
\left({ }_{a} D_{x}^{\alpha}\right)\left[f\left(\lambda x^{\alpha}\right) \otimes g\left(\mu x^{\alpha}\right)\right]=\left({ }_{a} D_{x}^{\alpha}\right)\left[f\left(\lambda x^{\alpha}\right)\right] \otimes g\left(\mu x^{\alpha}\right)+f\left(\lambda x^{\alpha}\right) \otimes \\
\left({ }_{a} D_{x}^{\alpha}\right)\left[g\left(\mu x^{\alpha}\right)\right] .(11)
\end{array}
$$

\section{Results and discussions}

Here we mainly discuss three types of fractional differential equations which can be transformed into separable variables

Theorem 3.1: Let $0<\alpha \leq 1$, then the first order homogeneous fractional differential equation

$$
{ }_{0} D_{x}^{\alpha}[y]=g\left(y \otimes\left(\frac{1}{\Gamma(\alpha+1)} x^{\alpha}\right)^{\otimes-1}\right)
$$

can be transformed into separable variables.

Proof Let $u=y \otimes\left(\frac{1}{\Gamma(\alpha+1)} x^{\alpha}\right)^{\otimes-1}$, then $y=u \otimes \frac{1}{\Gamma(\alpha+1)} x^{\alpha}$. Using product rule for fractional derivatives yields

$$
{ }_{0} D_{x}^{\alpha}[y]=u+\frac{1}{\Gamma(\alpha+1)} x^{\alpha} \otimes{ }_{0} D_{x}^{\alpha}[u]
$$

And hence,

$$
{ }_{0} D_{x}^{\alpha}[u]=(g(u)-u) \otimes\left(\frac{1}{\Gamma(\alpha+1)} x^{\alpha}\right)^{\otimes-1} .
$$

Since Eq. (14) is a separable variable fractional differential equation, the desired result holds.

Q.e.d.

Theorem 3.2: If $0<\alpha \leq 1, A, B, C$ are real numbers, then the first order fractional differential equation

$$
{ }_{0} D_{x}^{\alpha}[y]=h\left(\frac{A}{\Gamma(\alpha+1)} x^{\alpha}+B y+C\right)
$$

can be transformed into separable variables.

Proof Let $u=\frac{A}{\Gamma(\alpha+1)} x^{\alpha}+B y+C$, then

$$
{ }_{0} D_{x}^{\alpha}[u]=A+B \cdot{ }_{0} D_{x}^{\alpha}[y] \text {. }
$$


Therefore,

$$
{ }_{0} D_{x}^{\alpha}[u]=A+B h(u)
$$

Eq. (17) is a separable variable fractional differential equation, and hence the desired result holds.

Q.e.d.

Theorem 3.3: Assume that $0<\alpha \leq 1$ and $A, B, C, D, E, F$ are real numbers, then the first order fractional differential equation

$$
{ }_{0} D_{x}^{\alpha}[y]=f\left(\left(\frac{A}{\Gamma(\alpha+1)} x^{\alpha}+B y+C\right) \otimes\left(\frac{D}{\Gamma(\alpha+1)} x^{\alpha}+E y+F\right)^{\otimes-1}\right) .
$$

can be transformed into separable variables.

Proof Case 1. If $C=F=0$, then

$$
\begin{aligned}
&{ }_{0} D_{x}^{\alpha}[y]=f\left(\left(\frac{A}{\Gamma(\alpha+1)} x^{\alpha}+B y\right) \otimes\left(\frac{D}{\Gamma(\alpha+1)} x^{\alpha}+E y\right)^{\otimes-1}\right) \\
&=f\left(\left(A+B y \otimes\left(\frac{1}{\Gamma(\alpha+1)} x^{\alpha}\right)^{\otimes-1}\right) \otimes\left(D+E y \otimes\left(\frac{1}{\Gamma(\alpha+1)} x^{\alpha}\right)^{\otimes-1}\right)^{\otimes-1}\right) \\
&=g\left(y \otimes\left(\frac{1}{\Gamma(\alpha+1)} x^{\alpha}\right)^{\otimes-1}\right) .
\end{aligned}
$$

Thus, by Theorem 3.1, the desired result holds.

Case 2. If $C^{2}+F^{2} \neq 0$ and $\left|\begin{array}{ll}A & B \\ D & E\end{array}\right| \neq 0$. Then the system of equations

has a unique solution

$$
\left\{\begin{array}{l}
\frac{A}{\Gamma(\alpha+1)} x^{\alpha}+B y+C=0 \\
\frac{D}{\Gamma(\alpha+1)} x^{\alpha}+E y+F=0
\end{array}\right.
$$

$$
\left\{\begin{array}{c}
\frac{1}{\Gamma(\alpha+1)} x^{\alpha}=\lambda \\
y=\mu
\end{array}\right.
$$

Let $\left\{\begin{aligned} \frac{1}{\Gamma(\alpha+1)} X^{\alpha} & =\frac{1}{\Gamma(\alpha+1)} x^{\alpha}-\lambda \\ Y & =y-\mu\end{aligned}\right.$, then $\left\{\begin{aligned} \frac{A}{\Gamma(\alpha+1)} X^{\alpha}+B Y & =0 \\ \frac{D}{\Gamma(\alpha+1)} X^{\alpha}+E Y & =0\end{aligned}\right.$, and hence

$$
\begin{gathered}
{ }_{0} D_{X}^{\alpha}[Y]=f\left(\left(A+B Y \otimes\left(\frac{1}{\Gamma(\alpha+1)} X^{\alpha}\right)^{\otimes-1}\right) \otimes\left(D+E Y \otimes\left(\frac{1}{\Gamma(\alpha+1)} X^{\alpha}\right)^{\otimes-1}\right)^{\otimes-1}\right) \\
=g\left(Y \otimes\left(\frac{1}{\Gamma(\alpha+1)} X^{\alpha}\right)^{\otimes-1}\right) .
\end{gathered}
$$

Therefore, by Theorem 3.1, the desired result holds.

Case 3. If $C^{2}+F^{2} \neq 0$ and $\left|\begin{array}{ll}A & B \\ D & E\end{array}\right|=0$. There are three situations to discuss: (i) $A=B=0$, then

Eq. (18) becomes ${ }_{0} D_{x}^{\alpha}[y]=f\left(C \otimes\left(\frac{D}{\Gamma(\alpha+1)} x^{\alpha}+E y+F\right)^{\otimes-1}\right)$; (ii) $D=E=0$, then Eq. (18) becomes ${ }_{0} D_{x}^{\alpha}[y]=f\left(\frac{1}{F} \otimes\left(\frac{A}{\Gamma(\alpha+1)} x^{\alpha}+B y+C\right)\right)$; (iii) $\frac{A}{D}=\frac{B}{E}=k$, let $u=$ 
$\frac{D}{\Gamma(\alpha+1)} x^{\alpha}+E y$, then ${ }_{0} D_{x}^{\alpha}[u]=D+E_{0} D_{x}^{\alpha}[y]=D+E f\left((k u+C) \otimes(u+F)^{\otimes-1}\right)=$ $g(u)$ which is a separable variable fractional differential equation.

\section{Examples}

For the three types of first order fractional differential equations discussed in this article, we will give some examples and find their solutions.

Example 4.1: Consider the first order $1 / 3$ - fractional differential equation

$$
{ }_{0} D_{x}^{1 / 3}[y]=3 y \otimes\left(\frac{1}{\Gamma(4 / 3)} x^{1 / 3}\right)^{\otimes-1}+2\left(y \otimes\left(\frac{1}{\Gamma(4 / 3)} x^{1 / 3}\right)^{\otimes-1}\right)^{\otimes 2} .
$$

Let $u=y \otimes\left(\frac{1}{\Gamma(4 / 3)} x^{1 / 3}\right)^{\otimes-1}$, then $y=u \otimes \frac{1}{\Gamma(4 / 3)} x^{1 / 3}$. By product rule for fractional derivatives, we have ${ }_{0} D_{x}^{1 / 3}[y]=u+\frac{1}{\Gamma(4 / 3)} x^{1 / 3} \otimes{ }_{0} D_{x}^{1 / 3}[u]$. Thus,

$$
{ }_{0} D_{x}^{1 / 3}[u]=\left(\frac{1}{\Gamma(4 / 3)} x^{1 / 3}\right)^{\otimes-1} \otimes\left(2 u+2 u^{\otimes 2}\right) \text {. }
$$

Hence, we obtain the general solution of Eq. (23)

$$
y=c\left(\frac{1}{\Gamma(4 / 3)} x^{1 / 3}\right)^{\otimes 2} \otimes\left(\frac{1}{\Gamma(4 / 3)} x^{1 / 3}+y\right),
$$

and the particular solution $y=-\frac{1}{\Gamma(4 / 3)} x^{1 / 3}$, where $c$ is a constant.

Example 4.2: We study the first order $1 / 2$ - fractional differential equation

$$
{ }_{0} D_{x}^{1 / 2}[y]=\left(\frac{1}{\Gamma(3 / 2)} x^{1 / 2}+y\right)^{\otimes 2} \text {. }
$$

Let $v=\frac{1}{\Gamma(3 / 2)} x^{1 / 2}+y$, then ${ }_{0} D_{x}^{1 / 2}[v]=1+{ }_{0} D_{x}^{1 / 2}[y]$. So,

$$
{ }_{0} D_{x}^{1 / 2}[v]=1+v^{\otimes 2} \text {. }
$$

And hence, the general solution of Eq. (26) is

where $c$ is a constant.

$$
\frac{1}{\Gamma(3 / 2)} x^{1 / 2}+y=\tan _{1 / 2}\left(\frac{1}{\Gamma(3 / 2)} x^{1 / 2}+c\right)
$$

Example 4.3: Consider the first order $1 / 4$ - fractional differential equation

$$
{ }_{0} D_{x}^{1 / 4}[y]=\left(\frac{1}{\Gamma(5 / 4)} x^{1 / 4}-y+1\right) \otimes\left(\frac{1}{\Gamma(5 / 4)} x^{1 / 4}+y-3\right)^{\otimes-1} .
$$

Let $\left\{\begin{aligned} \frac{1}{\Gamma(5 / 4)} X^{1 / 4} & =\frac{1}{\Gamma(5 / 4)} x^{1 / 4-1} \\ Y & =y-2\end{aligned}\right.$, then we obtain

$$
{ }_{0} D_{X}^{1 / 4}[Y]=\left(\frac{1}{\Gamma(5 / 4)} X^{1 / 4}-Y\right) \otimes\left(\frac{1}{\Gamma(5 / 4)} X^{1 / 4}+Y\right)^{\otimes-1} .
$$

Let $u=Y \otimes\left(\frac{1}{\Gamma(5 / 4)} X^{1 / 4}\right)^{\otimes-1}$, then $Y=u \otimes \frac{1}{\Gamma(5 / 4)} X^{1 / 4}$. Thus,

$$
{ }_{0} D_{X}^{1 / 4}[u]=\left(\frac{1}{\Gamma(5 / 4)} X^{1 / 4}\right)^{\otimes-1} \otimes\left(1-2 u-u^{\otimes 2}\right) \otimes(1+u)^{\otimes-1} .
$$

Therefore, the general solution of Eq. (31) is 


$$
Y^{\otimes 2}+\frac{2}{\Gamma(5 / 4)} X^{1 / 4} \otimes Y-\left(\frac{1}{\Gamma(5 / 4)} X^{1 / 4}\right)^{\otimes 2}=c_{1}
$$

Hence, we obtain the general solution of Eq. (29)

$$
y^{\otimes 2}+\frac{2}{\Gamma(5 / 4)} x^{1 / 4} \otimes y-\left(\frac{1}{\Gamma(5 / 4)} x^{1 / 4}\right)^{\otimes 2}-6 y-\frac{2}{\Gamma(5 / 4)} x^{1 / 4}=c
$$

where $c$ is a constant.

\section{Conclusions}

As mentioned above, we can obtain the solutions of three types of first order fractional differential equations studied in this paper by using product rule for fractional derivatives. In fact, the application of product rule is extensive, and can be used to easily solve many fractional differential equations. On the other hand, our results are generalizations of classical first order differential equations which can be transformed into separable variables. In the future, we will use the Jumarie's modified R-L fractional derivatives and the new multiplication defined in this article to expand our research topics to the problems of applied mathematics and fractional calculus.

\section{References}

1. A. Bueno-Orovio, D. Kay, V. Grau, B. Rodriguez, K. Burrage, Fractional diffusion models of cardiac electrical propagation: role of structural heterogeneity in dispersion of repolarization, Tech. Rep. OCCAM 13/35, Oxford Centre for Collaborative Applied Mathematics, Oxford, UK, (2013).

2. D. Cafagna, G. Grassi, Observer-based projective synchronization of fractional systems via a scalar signal: Application to hyperchaotic Rössler systems, Nonlinear Dyn. 68 (1-2), (2012), pp. 117-128.

3. R. Caponetto, G. Maione, A. Pisano, M.M.R. Rapaic, ' E. Usai, Analysis and shaping of the self-sustained oscillations in relay controlled fractional-order systems, Fract. Calculus Appl. Anal. 16 (1), (2013), pp. 93-108.

4. R. Garra, Fractional-calculus model for temperature and pressure waves in fluid-saturated porous rocks, Phys. Rev. E 84 (2011) 036605.

5. R. L. Magin, Fractional calculus in bioengineering, in: 2012 13th International Carpathian Control Conference, ICCC 2012, (2012).

6. R. L. Magin, Fractional calculus models of complex dynamics in biological tissues, Comput. Math. Appl. 59 (5), (2010), pp.1586-1593.

7. J. Tenreiro Machado, P. Stefanescu, O. Tintareanu, D. Baleanu, Fractional calculus analysis of the cosmic microwave background, Romanian Rep. Phys. 65 (1), (2013), pp. 316-323.

8. J. T. Machado, V. Kiryakova, F. Mainardi, Recent history of fractional calculus, Commun. Nonlinear Sci. Numer. Simul. 16 (3), (2011), pp.1140-1153.

9. C. -H. Yu, Differential properties of fractional functions, International Journal of Novel Research in Interdisciplinary Studies, Vol.7, No. 5, (2020), pp.1-14.

10. C. - H. Yu, Fractional Clairaut's differential equation and its application, International Journal of Computer Science and Information Technology Research, Vol. 8, Issue 4, (2020), pp. 46-49.

11. C. -H. Yu, Separable fractional differential equations, International Journal of Mathematics and Physical Sciences Research, Vol. 8, Issue 2, (2020), pp. 30-34. 
12. C. - H. Yu, Fractional derivatives of some fractional functions and their applications, Asian Journal of Applied Science and Technology, Vol. 4, Issue 1, (2020), pp. 147-158.

13. C. -H. $\mathrm{Yu}, \mathrm{A}$ study on fractional RLC circuit, International Research Journal of Engineering and Technology, Vol. 7, Issue 8, (2020), pp. 3422-3425.

14. C. - H. $\mathrm{Yu}$, Method for evaluating fractional derivatives of fractional functions, International Journal of Scientific Research in Science, Engineering and Technology, Volume 7, Issue 4, (2020), pp. 286-290.

15. C. - H. Yu, Some fractional differential formulas, International Journal of Novel Research in Physics Chemistry \& Mathematics, Volume 7, Issue 3, (2020), pp. 1-4.

16. C. - H. Yu, Integral form of particular solution of nonhomogeneous linear fractional differential equation with constant coefficients, International Journal of Novel Research in Engineering and Science, Vol. 7, Issue 2, (2020), pp. 1-9.

17. C. -H. Yu, A study of exact fractional differential equations, International Journal of Interdisciplinary Research and Innovations, Vol. 8, Issue 4, (2020), pp. 100-105.

18. C. -H. Yu, Research on first order linear fractional differential equations, International Journal of Engineering Research and Reviews, Vol. 8, Issue 4, (2020), pp. 33-37.

19. C. -H. Yu, Method for solving fractional Bernoulli's differential equation, International Journal of Science and Research, Vol. 9, Issue 11, (2020), pp. 1684-1686.

20. C. - H. Yu, Using integrating factor method to solve some types of fractional differential equations, World Journal of Innovative Research, Vol. 9, Issue 5, (2020), pp. 161-164.

21. K. S. Miller and B. Ross, An Introduction to the Fractional Calculus and Fractional Differential Equations, John Wiley \& Sons, Inc., New York, (1993).

22. I. Podlubny, Fractional Differential Equations, Academic Press, San Diego (1999).

23. K. B. Oldham and J. Spanier, The Fractional Calculus: Theory and Application of Differentiation and Integration to Arbitrary Order, Academic Press, New York, (1974).

24. S. Das, Functional Fractional Calculus, 2nd ed. Springer-Verlag, (2011).

25. G. Jumarie, Modified Riemann-Liouville derivative and fractional Taylor series of nondifferentiable functions further results, Computers \& Mathematics with Applications, Vol. 51, No. 9, (2006), pp.1367-1376.

26. U. Ghosh, S. Sengupta, S. Sarkar, and S. Das, Analytic solution of linear fractional differential equation with Jumarie derivative in term of Mittag-Leffler function, American Journal of Mathematical Analysis, Vol. 3, No. 2, (2015), pp. 32-38.

27. J. C. Prajapati, Certain properties of Mittag-Leffler function with argument $x^{\alpha}, \alpha>0$, Italian Journal of Pure and Applied Mathematics, Vol. 30, (2013), pp. 411-416. 